\title{
First report of the root-knot nematode (Meloidogyne graminicola) in Madagascar rice fields
}

\author{
Elodie Chapuis $^{1,2}$ (1) $\cdot$ Guillaume Besnard $^{3} \cdot$ Simon Andrianasetra $^{4}$. \\ Mbolarinosy Rakotomalala ${ }^{4}$ • Hieu Trang Nguyen $^{5}$ • Stéphane Bellafiore ${ }^{1,5}$
}

Received: 4 June 2016 / Accepted: 28 October 2016/Published online: 4 November 2016

(C) Australasian Plant Pathology Society Inc. 2016

\begin{abstract}
Rice is the major crop produced in Madagascar and is infested by numerous plant-parasitic nematodes. An extensive list of plant-parasitic nematodes found in association with rice roots during a survey of 14 sites is presented. This is the first time that Meloidogyne graminicola has been detected in Madagascar, which is relevant information for future management of this economically important nematode pest of rice.
\end{abstract}

Keywords Rice $\cdot$ Plant parasitic nematode $\cdot$ Galls . Meloidogyne graminicola

Rice (Oryza sativa) is an essential food crop for a significant majority of the world's human population. Various pests and diseases constitute important constraints to successful crop production, and these include the plant-parasitic nematodes (PPN) that account for great crop-yield losses (Mantelin et al. 2016). Among the PPN, root-knot nematodes (genus

Elodie Chapuis

elodie.chapuis@ird.fr

1 UMR IPME (IRD, Université de Montpellier, CIRAD), 911 avenue Agropolis, BP 64501, 34394 Montpellier, Cedex 5, France

2 UMR PVBMT CIRAD-3P (CIRAD, Université de la Réunion), 7 Chemin de l'IRAT Ligne Paradis, 97410 Saint Pierre, La Réunion, France

3 CNRS-UPS-ENFA, EDB, UMR 5174, Bât. 4R1, 31062 Toulouse, Cedex 9, France

4 FOFIFA, Centre national de Recherche appliquée au développement rural, Centre Régional de Recherche au Nord-ouest, BP 289

Mahavoky avaratra, Mahajanga 401, Madagascar

5 IRD, LMI RICE, University of Science and Technology of Hanoi, Agricultural Genetics Institute, Hanoi, Vietnam
Meloidogyne) are considered to be the most damaging genus for numerous agricultural crops (Mantelin et al. 2016). In Asia, Meloidogyne graminicola is recognized as one of the most important pests of rice.

Second stage juveniles (J2) of M. graminicola penetrate the roots (Fig. 1), migrate to the vascular cylinder and instigate the formation of multinucleated giant cells. Infection by M. graminicola causes characteristic hook-shaped galls on the root tips of rice as has been seen in infected rice from South East Asian production areas (e.g., Thailand: Buangsuwon et al. 1971; India: Rao et al. 1970; Vietnam: Khuong 1983; Indonesia: Netscher and Erlan 1993), America (USA: Birchfield 1965; Colombia: Bastidas and Montealegre 1994; Brazil: Monteiro and Barbosa Ferraz 1988). In Africa, it has been reported once in South Africa on grass roots of Paspalum sp. and was only identified according to morphological characteristics (Kleynhans 1991). To our knowledge, no study has ever been conducted in Madagascar on this root knot nematode.

Having an extensive distribution map of $M$. graminicola can be useful for future management strategies since synthetically-derived nematicides commonly used to control nematode pests are being continuously withdrawn from world markets (MBTOC 2010). Proper information on the nematodes present in the rice paddy is necessary before developing any environmentally sustainable management strategies. The purpose of this note is to report for the first time the presence of the rice root-knot nematode M. graminicola in Madagascar.

Between the $24^{\text {th }}$ of October 2014 and the $7^{\text {th }}$ of November 2014, 14 sites distributed along a NW/SE axis between the towns of Marovovay and Manakara (Madagascar) were surveyed (Fig. 2). In order to reflect the diversity of the local crop growth, the survey covered different fields within each site. In each field, two to six samples presenting what were thought to be the characteristic root galls of the genus Meloidogyne (Fig. 1) were 


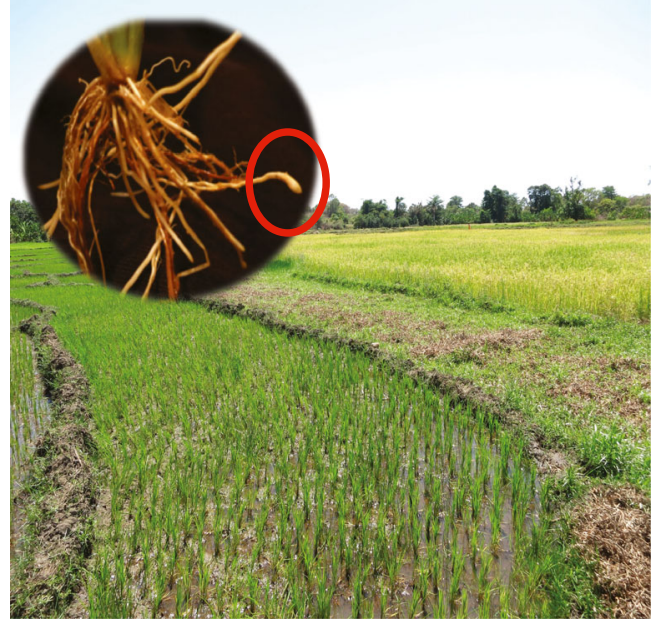

Fig. 1 Rice fields in Madagascar could present different plant development stages and are mostly rainfall agroecosystem. In foreground, a recently transplanted field and, in background, a field where rice plants are at the reproduction phase. The insert illustrates infected root systems showing gall formations with typical hook-shaped root tips that were subsequently find to contain Meloidogyne graminicola

collected, labelled and immediately put on ice in individual plastic bags. For each site, samples were pooled together then split into two subsamples. One subsample was sent to the IRD-IPME (Research Institute for the Development / Interactions PlantsMicroorganisms-Environment) and the other staying in Mahajanga at the FOFIFA (national centre of applied research for the rural development) Madagascar, as determined by the MTA (Material Transfer Agreement, $N^{\circ}$ Siroco: 304722/00) between France (IRD-IPME Montpellier) and Madagascar (FOFIFA-Mahajanga). Eggs and nematodes were recovered from infected roots using the hypochlorite extraction method with minor modifications (Bellafiore et al. 2015). Individual PPN were identified according to their characteristic stylet using a stereomicroscope and then manually transferred to a $0.2-\mathrm{mL}$ polymerase chain reaction (PCR) tube for extraction of total DNA in a proteinase $\mathrm{K}$ buffer. This manipulation was done only in IPME-Montpellier. In Montpellier, permanent slides of the juveniles were made, whereas the specimens were preserved in vials for the FOFIFA collection.

All PPN were then identified at the genus level by PCR followed by sequencing using ITS-1 amplification of rDNA genes as described previously in Bellafiore et al. (2015). For each individual PPN, the sequences were compared to other deposited ITS sequences in the GenBank database using standard nucleotide BLAST in April 2016. When a positive match to M. graminicola was observed, we confirmed the identification of this species using the SCAR-MgFw/Rev primers (Bellafiore et al. 2015) and the mitochondrial DNA marker 94R (Besnard et al. 2014). DNA amplicon obtained from mitochondrial DNA using the 94R primers was then subsequently gel-purified and sequenced as described above with the primer 94R-Fw (5'GAATGAAAACGATCAGAGACATG-3').

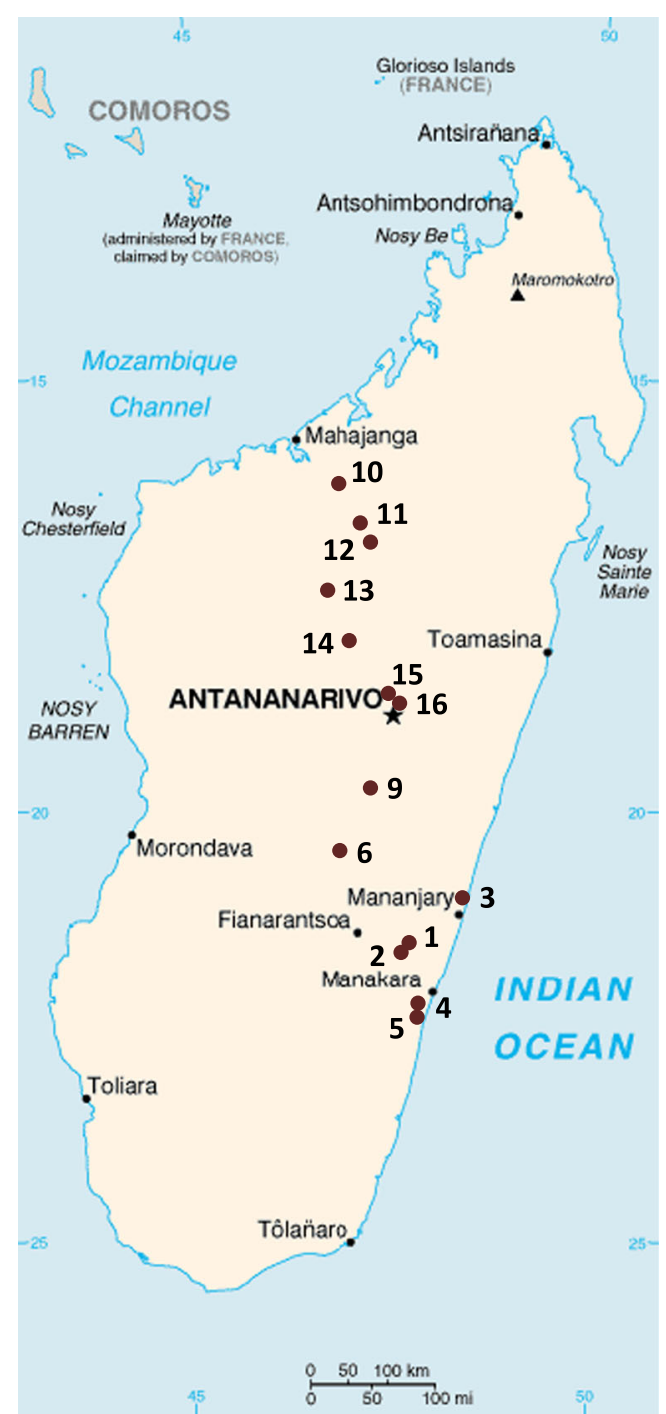

Fig. 2 Map of the sites sampled in October and November 2014, in Madagascar. Fourteen sites were visited

Ten of the 14 sites presented PPN in the root system of the collected rice plants (Table 1). Among them, we identified nematodes from seven of the nine most abundant worldwide root PPN genera described by Bridge et al. (2005). Only Hoplolaimus spp. and Paralongidorus spp. were not found. Using three molecular markers (ITS, SCAR-Mg and the mitochondrial 94R), we confirmed the presence of M. graminicola populations in three independent sites $\left(\mathrm{N}^{\mathrm{o}} 1,5\right.$, 6; Table 1). Interestingly, we also found Meloidogyne spp. in two other sites $\left(\mathrm{N}^{\mathrm{o}} 3\right.$ and 6 ; Table 1). ITS sequences indicate that these accessions are closely related to $M$. graminicola (three SNPs + one indel from a 182-bp sequence and six SNPs + seven indels from a 339-bp sequence, respectively). We did not succeed in amplifying the markers SCAR and 94R in those two populations, suggesting that they are distinct from M. graminicola. Isolated juveniles (second stage) M. graminicola individuals from each site presented (i.e. 17 
Table 1 The 14 sites sampled, and their GPS coordinates (using the Geodetic Reference System WGS 84)

\begin{tabular}{|c|c|c|c|c|c|c|}
\hline Site & Latitude & Longitude & Sample & Accession number & Specimen voucher & PPN \\
\hline \multirow[t]{5}{*}{1} & \multirow[t]{5}{*}{-21.381190} & \multirow[t]{5}{*}{47.914980} & $1-1$ & KX372251 & FOFIFA-MG-1-1 & M. graminicola \\
\hline & & & $1-2$ & KX372252 & FOFIFA-MG-1-2 & M. graminicola \\
\hline & & & $1-3$ & KX372253 & FOFIFA-MG-1-3 & M. graminicola \\
\hline & & & $1-4$ & KX372254 & FOFIFA-MG-1-4 & M. graminicola \\
\hline & & & $1-5$ & KX372255 & FOFIFA-MG-1-5 & M. graminicola \\
\hline 2 & -21.245628 & 48.322455 & $2-1$ & KX372256 & FOFIFA-MG-2-1 & Aphelenchoides spp. \\
\hline 3 & -21.496380 & 47.966200 & $3-1$ & KX372257 & FOFIFA-MG-3-1 & Meloidogyne spp. \\
\hline 4 & -22.140906 & 48.018986 & No sample & - & - & No \\
\hline \multirow[t]{2}{*}{5} & \multirow[t]{2}{*}{-22.032891} & \multirow[t]{2}{*}{47.972814} & $5-1$ & KX372258 & FOFIFA-MG-5-1 & M. graminicola \\
\hline & & & $5-2$ & KX372259 & FOFIFA-MG-5-2 & M. graminicola \\
\hline \multirow[t]{5}{*}{6} & \multirow[t]{5}{*}{-20.565795} & \multirow[t]{5}{*}{47.232783} & $6-1$ & KX372260 & FOFIFA-MG-6-1 & Helicotylenchus spp. \\
\hline & & & $6-2$ & KX372261 & FOFIFA-MG-6-2 & Xiphinema spp. \\
\hline & & & $6-3$ & KX372262 & FOFIFA-MG-6-3 & Meloidogyne spp. \\
\hline & & & $6-4$ & KX372263 & FOFIFA-MG-6-4 & Xiphinema spp. \\
\hline & & & $6-5$ & KX372264 & FOFIFA-MG-6-5 & M. graminicola \\
\hline 9 & -19.645043 & 47.305249 & No sample & - & - & No \\
\hline 10 & -16.563188 & 47.098087 & 10.1 & - & - & Unidentified \\
\hline 11 & -16.358330 & 47.001540 & No sample & - & - & No \\
\hline 12 & -16.132876 & 46.747818 & No sample & - & - & No \\
\hline 13 & -16.973774 & 46.948438 & $13-1$ & - & - & Unidentified \\
\hline \multirow[t]{2}{*}{14} & \multirow[t]{2}{*}{-18.265880} & \multirow[t]{2}{*}{47.172730} & $14-1$ & KX372265 & FOFIFA-MG-14-1 & Pratylenchus spp. \\
\hline & & & $14-2$ & KX372266 & FOFIFA-MG-14-2 & Helicotylenchus spp. \\
\hline \multirow[t]{2}{*}{15} & \multirow[t]{2}{*}{-18.755776} & \multirow[t]{2}{*}{47.619490} & $15-1$ & KX372267 & FOFIFA-MG-15-1 & Heterodera spp. \\
\hline & & & $15-3$ & KX372268 & FOFIFA-MG-15-3 & Heterodera spp. \\
\hline \multirow[t]{2}{*}{16} & \multirow[t]{2}{*}{-18.756507} & \multirow[t]{2}{*}{47.705949} & $16-1$ & KX372269 & FOFIFA-MG-16-1 & Hirschmanniella spp. \\
\hline & & & $16-2$ & KX372270 & FOFIFA-MG-16-2 & Hirschmanniella spp. \\
\hline
\end{tabular}

The last column contains the PPN (plant parasitic nematode) identified: Meloidogyne graminicola (M. graminicola) was found in three sites. "No sample" meant that the site was prospected but since we did not find any galls we did not collect any samples. The accession numbers and the specimen vouchers refer to GenBank inventory

individuals) an average body length of 487.5 millimetres (ranging from 434 to $516 \mathrm{~mm}$ ) with a standard error of 1.7 ; and an average stylet length of $12.6 \mathrm{~mm}(12.2$ to $13.2 \mathrm{~mm})$ with a standard error of $=0.1$.

Both M. graminicola populations as well as the two M. graminicola related species identified in this survey were isolated from fields present in the South East region of Antsirabe. In this region, rice is mainly grown under lowland and irrigation conditions, two water regimes favourable to M. graminicola that could explain the presence of this species in this part of the island. To our knowledge, this report represents the first record of M. graminicola on rice in Madagascar and Africa. As mentioned above, M. graminicola has only been found once in South Africa (Kleynhans 1991) but this was on another plant host and in other environmental conditions. We hereby propose a possible hypothesis: rice in Madagascar is likely to have come from South East Asia and not from Africa since the first Malagasy people migrated from SE Asia (Indonesia and Malaysia: Dewar and Wright 1993; Perrier de la Bâthie 1931) bringing with them their homeland crops. Moreover, these would have been distributed from the eastern coast ports (S. Andrianasetra, pers. observ.) where we also found M. graminicola. More genetic population studies and surveys in Madagascar and Africa are required to confirm or infirm this hypothesis.

\section{References}

Bastidas H, Montealegre SFA (1994) Aspectos generales de la nueva enfermedad del arroz llamada entorchamiento. Arroz 43:30-35

Bellafiore S, Jougla C, Chapuis É, Besnard G, Suong M, Nguyen Vu P, De Waele D, Gantet P, Xuyen Ngo T (2015) Intraspecific variability of the facultative meiotic parthenogenetic root-knot nematode (Meloidogyne graminicola) from rice fields in Vietnam. C R Biol 338:471-483 
Besnard G, Jühling F, Chapuis É, Zedane L, Lhuillier É, Mateille T, Bellafiore S (2014) Fast assembly of the mitochondrial genome of a plant parasitic nematode (Meloidogyne graminicola) using next generation sequencing. C R Biol 337:295-301

Birchfield W (1965) Host parasite relations and host range studies of a new Meloidogyne species in southern USA. Phytopathology 55:1359-1361

Bridge J, Plowright RA, Peng D (2005) Nematode parasites of rice. In: Luc M, Sikora RA, Bridge J (eds) Plant parasitic nematodes in subtropical and tropical agriculture. CABI Bioscience, Wallingford, pp 87-130

Buangsuwon D, Tonboon-ek P, Rujirachoon G, Braun AJ, Taylor AL (1971) Nematodes. In: Rice Diseases and Pests of Thailand. English Edition. Rice Protection Research Centre, Ministry of Agriculture. Thailand, pp. 61-67

Dewar RE, Wright HT (1993) The culture history of Madagascar. J World Prehist 7:417-466

Khuong NB (1983) Plant-parasitic nematodes of South Viet Nam. J Nematol 15:319-323

Kleynhans KP (1991) The root-knot nematodes of South Africa. Technical Communication, Department of Agricultural Development, South Africa, No. 231:61, pp. 136
Mantelin S, Bellafiore S, Kyndt T (2016) Meloidogyne graminicola a major threat to rice agriculture. Mol Plant Pathol. doi:10.1111 /mpp.12394

MBTOC (2010) Report of the Methyl Bromide Technical Options Committee (MBTOC) 2010 Assessment. Montreal protocol on substances that deplete the ozone layer, Nairobi, Kenya: United Nations Environment Programme (UNEP)

Monteiro AR, Barbosa Ferraz LCC (1988) First record and preliminary information on the host range of Meloidogyne graminicola in Brazil. Nematol Brasil 12:149-150

Netscher C, Erlan X (1993) A root-knot nematode, Meloidogyne graminicola, parasitic on rice in Indonesia. Afro-Asian J Nematol 3:90-95

Perrier de la Bâthie H (1931) Les Plantes introduites à Madagascar. Rev Bot Appl Agric Trop 11:833-837

Rao YS, Israel P, Biswas H (1970) Weed and rotation crop plants as hosts for the rice root-knot nematode, Meloidogyne graminicola (Golden and Birchfield). Oryza 7:137-142 